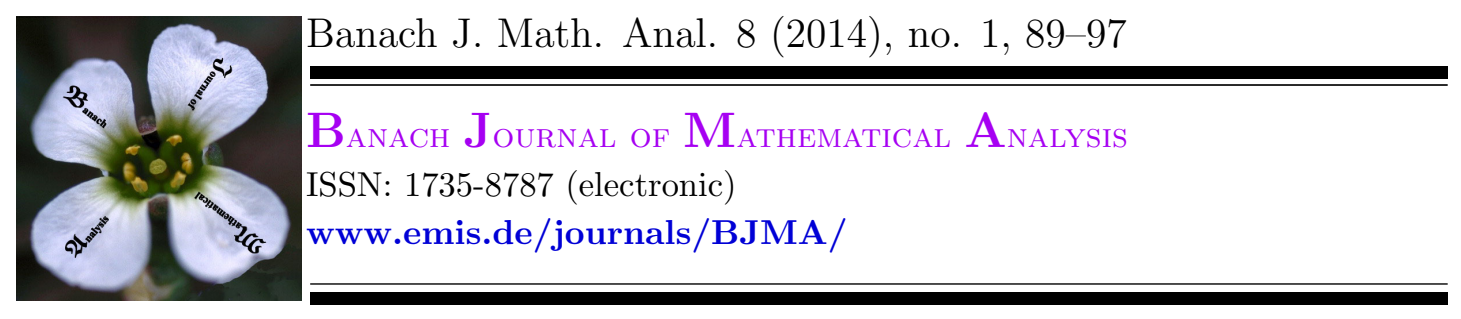

\title{
ORTHONORMAL BASES AND A STRUCTURE OF FINITE DIMENSIONAL NORMED LINEAR SPACES
}

\author{
RYOTARO TANAKA ${ }^{1}$ AND KICHI-SUKE SAITO ${ }^{2 *}$ \\ Communicated by D. Bakić
}

\begin{abstract}
In this paper, we consider orthonormal bases for finite dimensional normed linear spaces which are related to Birkhoff orthogonality. We also study the relationship between orthonormal bases and a structure of finite dimensional normed linear spaces.
\end{abstract}

\section{INTRODUCTION}

Throughout this paper, the term "normed linear space" always means a real normed linear space. Let $X$ be a normed linear space equipped with the norm $\|\cdot\|$. Then $B_{X}$ and $S_{X}$ are, respectively, the closed unit ball and unit sphere of $X$. If $A$ is a subset of a normed linear space, let $\langle A\rangle$ be the linear span of $A$.

Orthogonality is one of the most important concepts in the theory of inner product spaces. It appears in many fundamental theorems such as the Pythagorean theorem. However, when switching from the theory of inner product spaces to Banach space geometry, we cannot maintain the notion of orthogonality. Therefore, since 1934 generalized orthogonality types for normed linear spaces have been introduced and studied.

(i) Roberts $\perp_{R}$ : $x \perp_{R} y$ if $\|x+\alpha y\|=\|x-\alpha y\|$ for all $\alpha \in \mathbb{R}$ (cf. Roberts [13]).

(ii) Isosceles $\perp_{I}: x \perp_{I} y$ if $\|x+y\|=\|x-y\|$ (cf. James [9]).

(iii) Pythagorean $\perp_{P}: x \perp_{P}$ y if $\|x-y\|^{2}=\|x\|^{2}+\|y\|^{2}$ (cf. James [9]).

Date: Received: 8 April 2013; Accepted: 28 April 2013.

* Corresponding author.

2010 Mathematics Subject Classification. Primary 46B20; Secondary 46B25.

Key words and phrases. Birkhoff orthogonality, orthonormal basis, normed linear space. 
There are many other types of orthogonality in normed linear spaces such as Diminnie [8], Carlsson [5], T-orthogonality [15], and so on. The survey of AlonsoMartini-Wu [2] is a good starting point to study the history of generalized orthogonality.

In this paper, we focus on Birkhoff orthogonality especially. Let $X$ be a normed linear space and let $x, y \in X$. Then, recall that $x$ is said to be Birkhoff orthogonal to $y$, denoted by $x \perp_{B} y$, if $\|x+\alpha y\| \geq\|x\|$ for all $\alpha \in \mathbb{R}$. If $M$ and $N$ are two subspaces of $X$, then $M$ is said to be Birkhoff orthogonal to $N$, denoted by $M \perp_{B} N$, if $x \perp_{B} y$ for all $x \in M$ and all $y \in N$. In particular, $\langle\{x\}\rangle \perp_{B} N$ and $M \perp_{B}\langle\{y\}\rangle$ are simply denoted by $x \perp_{B} N$ and $M \perp_{B} y$, respectively. Recall that Birkhoff orthogonality is always homogeneous, that is, $x \perp_{B} y$ implies $\alpha x \perp_{B} \beta y$ for any real numbers $\alpha$ and $\beta$. However, Birkhoff orthogonality is not symmetric in general, that is, $x \perp_{B} y$ does not imply $y \perp_{B} x$. Indeed, a real normed linear space of dimension greater than or equal to three is an inner product space if and only if Birkhoff orthogonality is symmetric (Day [7], James [10]). More details about Birkhoff orthogonality can be found in $[4,6,7,9,10,11]$.

A norm $\|\cdot\|$ on $\mathbb{R}^{n}$ is said to be normal if it satisfies $\|\cdot\|_{1} \leq\|\cdot\| \leq\|\cdot\|_{\infty}$. Let $N N_{n}$ denote the set of all normal norms on $\mathbb{R}^{n}$. In the previous paper [16], we showed that every $n$-dimensional normed linear space is isometrically isomorphic to the space $\mathbb{R}^{n}$ endowed with a normal norm. If $n=2$, the result is due to Alonso [1]. This is essentially based on the following result of Day [6]: Every $n$-dimensional normed linear space $X$ has a basis $\left\{e_{1}, e_{2}, \ldots, e_{n}\right\} \subset S_{X}$ such that

$\left(B_{1}\right) e_{k} \perp_{B} M_{k}$ for all $k=1,2, \ldots, n$,

where $M_{k}=\left\langle\left\{e_{1}, \ldots, e_{k-1}, e_{k+1}, \ldots, e_{n}\right\}\right\rangle$. However, as mentioned in above, $e_{k} \perp_{B} M_{k}$ is not equivalent to $M_{k} \perp_{B} e_{k}$. So we consider a basis $\left\{e_{1}, e_{2}, \ldots, e_{n}\right\} \subset$ $S_{X}$ satisfying the following condition:

$\left(B_{2}\right) M_{k} \perp_{B} e_{k}$ for all $k=1,2, \ldots, n$,

A basis $\left\{e_{1}, e_{2}, \ldots, e_{n}\right\}$ for an $n$-dimensional normed linear space is said to be

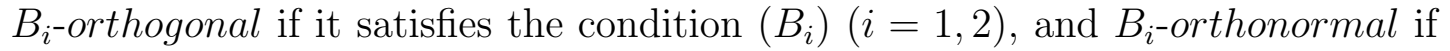
it is $B_{i}$-orthogonal and normal, that is, $\left\{e_{1}, e_{2}, \ldots, e_{n}\right\} \subset S_{X}$.

In this paper, we study $B_{1}$-orthonormal bases and $B_{2}$-orthonormal bases for finite dimensional normed linear spaces.

\section{Orthonormal BASES}

We first consider a characterization of orthonormal bases in terms of norms.

Proposition 2.1. Let $X$ be an $n$-dimensional normed linear space and $\left\{e_{1}, \ldots, e_{n}\right\}$ be a normal basis for $X$. Then, the following are equivalent:

(i) $\left\{e_{1}, e_{2}, \ldots, e_{n}\right\}$ is $B_{1}$-orthonormal.

(ii) The equation

$$
\left\|\sum_{i=1}^{n} a_{i} e_{i}\right\| \geq \max _{1 \leq i \leq n}\left|a_{i}\right|
$$

holds for all $\left(a_{1}, a_{2}, \ldots, a_{n}\right) \in \mathbb{R}^{n}$. 
Proof. We only note that for each $k=1,2, \ldots, n, e_{k} \perp_{B}\left[\left\{e_{i}\right\}_{i \neq k}\right]$ if and only if

$$
\left\|\sum_{i=1}^{n} a_{i} e_{i}\right\|=\left\|a_{k} e_{k}+\sum_{i \neq k} a_{i} e_{i}\right\| \geq\left|a_{k}\right|
$$

for all $\left(a_{1}, a_{2}, \ldots, a_{n}\right) \in \mathbb{R}^{n}$. Then one can easily check that (i) is equivalent to (ii).

Remark 2.2. Bases satisfying (ii) of Proposition 2.1 was considered by Khalil [12]. He mainly studied Schauder bases which satisfies (ii).

Proposition 2.3. Let $X$ be an $n$-dimensional normed linear space and $\left\{e_{1}, \ldots, e_{n}\right\}$ be a normal basis for $X$. Then, the following are equivalent:

(i) $\left\{e_{1}, e_{2}, \ldots, e_{n}\right\}$ is $B_{1}$-orthonormal.

(ii) The inequality

$$
\left\|\sum_{i=1}^{n} a_{i} e_{i}\right\| \geq \max _{1 \leq k \leq n}\left\|\sum_{i \neq k} a_{i} e_{i}\right\|
$$

holds for all $\left(a_{1}, a_{2}, \ldots, a_{n}\right) \in \mathbb{R}^{n}$.

Proof. Let $i=1,2, \ldots, n$. Since $\left[\left\{e_{1}, \ldots, e_{k-1}, e_{k+1}, \ldots, e_{n}\right\}\right] \perp_{B} e_{k}$ if and only if

$$
\left\|\sum_{i=1}^{n} a_{i} e_{i}\right\| \geq\left\|\sum_{i \neq k} a_{i} e_{i}\right\|
$$

for all $\left(a_{1}, a_{2}, \ldots, a_{n}\right) \in \mathbb{R}^{n}$, one has the equivalence of (i) and (ii).

We next consider the relationship between the conditions $\left(B_{1}\right)$ and $\left(B_{2}\right)$. Then, the following lemma is needed, which is an important characterization of the condition $\left(B_{2}\right)$. The proof is easy, and so is omitted.

Lemma 2.4. Let $\left\{e_{1}, e_{2}, \ldots, e_{n}\right\}$ be a $B_{2}$-orthonormal basis for an $n$-dimensional normed linear space. Then,

$$
\left\|\sum_{i=1}^{n} a_{i} e_{i}\right\|=\max \left\{\left\|\sum_{i \in A} a_{i} e_{i}\right\|: A \subset\{1,2, \ldots, n\}\right\}
$$

for all $\left(a_{1}, a_{2}, \ldots, a_{n}\right) \in \mathbb{R}^{n}$.

This means that if $\left\{e_{1}, e_{2}, \ldots, e_{n}\right\}$ is $B_{2}$-orthonormal, any natural projection on $X$ has norm 1 . Hence, $B_{2}$-orthonormal bases can be viewed as finite dimensional version of 1-unconditional bases.

From the preceding lemma, we immediately have the following result.

Theorem 2.5. Let $X$ be an $n$-dimensional real normed linear space. Suppose that $\left\{e_{1}, e_{2}, \ldots, e_{n}\right\}$ is a normal basis for $X$. Then, the following hold:

(i) If $\left\{e_{1}, e_{2}, \ldots, e_{n}\right\}$ is $B_{2}$-orthonormal, then it is $B_{1}$-orthonormal.

(ii) If $n=2$, then $\left\{e_{1}, e_{2}\right\}$ is $B_{2}$-orthonormal if and only if it is $B_{1}$-orthonormal. In symbols, $\left(B_{2}\right) \Rightarrow\left(B_{1}\right)$, and $\left(B_{2}\right) \Leftrightarrow\left(B_{1}\right)$ if $n=2$.

As seen in the following example, $\left(B_{1}\right)$ does not imply $\left(B_{2}\right)$ if $n \geq 3$. 
Example 2.6. Suppose that $n \geq 3$. Our purpose is to show that $\left(B_{1}\right)$ does not imply $\left(B_{2}\right)$ if $n \geq 3$. Let $\left\{e_{1}, e_{2}, \ldots, e_{n}\right\}$ be the standard unit vector basis for $\mathbb{R}^{n}$ and let $\|\cdot\|$ be the normalized norm on $\mathbb{R}^{n}$ defined by

$$
\left\|\left(a_{1}, a_{2}, \ldots, a_{n}\right)\right\|=\max \left\{\left|a_{1}\right|,\left|\sum_{k=1}^{n} a_{k}\right|, \max _{2 \leq i \leq n}\left|\sum_{k=1}^{n} \varepsilon_{i k} a_{k}\right|\right\}
$$

for all $\left(a_{1}, a_{2}, \ldots, a_{n}\right) \in \mathbb{R}^{n}$, where

$$
\varepsilon_{i k}=\left\{\begin{aligned}
1 & \text { if } k \neq i, \\
-1 & \text { if } k=i .
\end{aligned}\right.
$$

Then we have

$$
\begin{aligned}
\left|a_{i}\right| & =\left|\frac{1}{2}\left(\sum_{k=1}^{n} a_{k}-\sum_{k=1}^{n} \varepsilon_{i k} a_{k}\right)\right| \\
& \leq \frac{1}{2}\left(\left|\sum_{k=1}^{n} a_{k}\right|+\left|\sum_{k=1}^{n} \varepsilon_{i k} a_{k}\right|\right) \\
& \leq\left\|\left(a_{1}, a_{2}, \ldots, a_{n}\right)\right\|
\end{aligned}
$$

for each $i \geq 2$, and so

$$
\left\|\left(a_{1}, a_{2}, \ldots, a_{n}\right)\right\| \geq \max _{1 \leq i \leq n}\left|a_{i}\right|
$$

for all $\left(a_{1}, a_{2}, \ldots, a_{n}\right) \in \mathbb{R}^{n}$. Thus $\left\{e_{1}, e_{2}, \ldots, e_{n}\right\}$ satisfies $\left(B_{1}\right)$. However,

$$
\|(-1,1, \ldots, 1)\|=n-2<n-1=\|(0,1, \ldots, 1)\| .
$$

Therefore $\left\langle\left\{e_{k}\right\}_{k \geq 2}\right\rangle \not_{B} e_{1}$, and so $\left\{e_{1}, e_{2}, \ldots, e_{n}\right\}$ does not satisfy $\left(B_{2}\right)$.

\section{The NORM STRUCTURE}

In this section, we characterize the existence of orthonormal bases. The existence of $B_{1}$-orthonormal bases is characterized using normal norms.

Theorem 3.1. Let $X$ be an $n$-dimensional normed linear space. Then, $X$ has a $B_{1}$-orthonormal basis if and only if it is isometrically isomorphic to the space $\mathbb{R}^{n}$ endowed with a normal norm.

Proof. Suppose that $X$ has a $B_{1}$-orthonormal basis $\left\{e_{1}, e_{2}, \ldots, e_{n}\right\}$. Define a norm $\|\cdot\|_{0}$ on $\mathbb{R}^{n}$ by

$$
\left\|\left(a_{1}, a_{2}, \ldots, a_{n}\right)\right\|_{0}=\left\|\sum_{k=1}^{n} a_{k} e_{k}\right\|
$$

for all $\left(a_{1}, a_{2}, \ldots, a_{n}\right) \in \mathbb{R}^{n}$. Then, $\|\cdot\|_{0} \in N N_{n}$ by Proposition 2.1, and $X$ is isometrically isomorphic to the space $\left(\mathbb{R}^{n},\|\cdot\|_{0}\right)$.

Conversely, we assume that $X$ is isometrically isomorphic to the space $\mathbb{R}^{n}$ endowed with a regular norm $\|\cdot\|_{0}$. For each $i=1,2, \ldots, n$, let $e_{i}$ be the element of $X$ which identified with the $i$-th coordinate vector of $\mathbb{R}^{n}$. Then, we have

$$
\left\|\sum_{k=1}^{n} \alpha_{k} e_{k}\right\|=\left\|\left(\alpha_{1}, \alpha_{2}, \ldots, \alpha_{n}\right)\right\|_{0}
$$


for all $\left(\alpha_{1}, \alpha_{2}, \ldots, \alpha_{n}\right) \in \mathbb{R}^{n}$, and so

$$
\begin{aligned}
\left\|\sum_{k=1}^{n} \alpha_{k} e_{k}\right\| & =\left\|\left(\alpha_{1}, \alpha_{2}, \ldots, \alpha_{n}\right)\right\|_{0} \\
& \geq \max _{1 \leq i \leq n}\left|a_{i}\right|
\end{aligned}
$$

holds for all $\left(a_{1}, a_{2}, \ldots, a_{n}\right) \in \mathbb{R}^{n}$. Hence, $\left\{e_{1}, e_{2}, \ldots, e_{n}\right\}$ is a $B_{1}$-orthonormal basis for $X$ by Proposition 2.1. The proof is complete.

Remark 3.2. As was mentioned in introduction, Day [6] showed that every finite dimensional normed linear space has a $B_{1}$-orthonormal basis.

We next consider a characterization of $B_{2}$-orthonormal bases. For this purpose, we introduce the notion of regular norm. We recall some about norms on $\mathbb{R}^{n}$.

Definition 3.3. A norm $\|\cdot\|$ on $\mathbb{R}^{n}$ is said to be absolute if

$$
\left\|\left(a_{1}, a_{2}, \ldots, a_{n}\right)\right\|=\left\|\left(\left|a_{1}\right|,\left|a_{2}\right|, \ldots,\left|a_{n}\right|\right)\right\|
$$

for all $\left(a_{1}, a_{2}, \ldots, a_{n}\right) \in \mathbb{R}^{n}$, and normalized if

$$
\|(1,0, \ldots, 0)\|=\|(0,1,0, \ldots, 0)\|=\|(0, \ldots, 0,1)\|=1 .
$$

Let $A N_{n}$ denote the family of all absolute normalized norms on $\mathbb{R}^{n}$.

The following is an important characterization of absolute norm. The proof can be found in [3, Proposition IV.1.1] (see, also [14, Lemma 4.1]).

Lemma 3.4. A norm $\|\cdot\|$ on $\mathbb{R}^{n}$ is absolute if and only if it is monotone, that is, if $\left|a_{k}\right| \leq\left|b_{k}\right|$ for all $k=1,2, \ldots, n$ then $\left\|\left(a_{1}, a_{2}, \ldots, a_{n}\right)\right\| \leq\left\|\left(b_{1}, b_{2}, \ldots, b_{n}\right)\right\|$.

We now introduce regular norms on $\mathbb{R}^{n}$.

Definition 3.5. A norm $\|\cdot\|$ on $\mathbb{R}^{n}$ is said to be regular if it is normalized and

$$
\left\|\left(a_{1}, a_{2}, \ldots, a_{n}\right)\right\| \geq \max _{1 \leq k \leq n}\left\|\left(a_{1}, \ldots, a_{k-1}, 0, a_{k+1}, \ldots, a_{n}\right)\right\|
$$

for all $\left(a_{1}, a_{2}, \ldots, a_{n}\right) \in \mathbb{R}^{n}$. Let $R N_{n}$ denote the family of all regular norms on $\mathbb{R}^{n}$.

Regular norms have a characterization analogous to Lemma 3.4. To see this, we introduce the notion of the $\Omega_{i}$-quadrant. For each $n \geq 2$, define a $2^{n-1} \times n$ matrix $R_{n}^{+}$by the formula

$$
R_{2}^{+}=\left(\begin{array}{rr}
1 & 1 \\
1 & -1
\end{array}\right) \text { and } R_{n}^{+}=\left(\begin{array}{c|c}
1 & \\
\vdots & R_{n-1}^{+} \\
1 & \\
\hline 1 & \\
\vdots & -R_{n-1}^{+} \\
1 &
\end{array}\right) \quad(n \geq 3)
$$


Fix a positive integer $n \geq 2$. Let

$$
R_{n}^{+}=\left(\begin{array}{c}
\theta_{1} \\
\theta_{2} \\
\vdots \\
\theta_{2^{n-1}}
\end{array}\right) .
$$

Then, the $\Omega_{i}$-quadrant of $\mathbb{R}^{n}$ is given by

$$
\Omega_{i}=\theta_{i} \cdot \mathbb{R}_{+}^{n}=\left\{\theta_{i} \cdot x: x \in \mathbb{R}_{+}^{n}\right\},
$$

where $\mathbb{R}_{+}$is the set of all nonnegative real numbers, and $\theta_{i} \cdot x$ denotes the pointwise product of $\theta_{i}$ and $x$. For example, if $n=3$,

$$
R_{3}^{+}=\left(\begin{array}{rrr}
1 & 1 & 1 \\
1 & 1 & -1 \\
1 & -1 & -1 \\
1 & -1 & 1
\end{array}\right)
$$

and

$$
\begin{aligned}
& \Omega_{1}=\left\{(x, y, z) \in \mathbb{R}^{3}: x, y, z \geq 0\right\}, \\
& \Omega_{2}=\left\{(x, y,-z) \in \mathbb{R}^{3}: x, y, z \geq 0\right\}, \\
& \Omega_{3}=\left\{(x,-y,-z) \in \mathbb{R}^{3}: x, y, z \geq 0\right\}, \\
& \Omega_{4}=\left\{(x,-y, z) \in \mathbb{R}^{3}: x, y, z \geq 0\right\} .
\end{aligned}
$$

The following is an analog of Lemma 3.4 for regular norms.

Theorem 3.6. A normalized norm $\|\cdot\|$ on $\mathbb{R}^{n}$ is regular if and only if it is semi-monotone, that is,

$$
\left\|\left(a_{1}, a_{2}, \ldots, a_{n}\right)\right\| \leq\left\|\left(b_{1}, b_{2}, \ldots, b_{n}\right)\right\|
$$

whenever $\left(a_{1}, a_{2}, \ldots, a_{n}\right),\left(b_{1}, b_{2}, \ldots, b_{n}\right) \in \Omega_{i}$ for some $i$ and $\left|a_{k}\right| \leq\left|b_{k}\right|$ for all $k=1,2, \ldots, n$, or equivalently, whenever $\left(a_{1}, a_{2}, \ldots, a_{n}\right),\left(b_{1}, b_{2}, \ldots, b_{n}\right)$ satisfies $a_{k} b_{k} \geq 0$ and $\left|a_{k}\right| \leq\left|b_{k}\right|$ for all $k=1,2, \ldots, n$.

Proof. If $\|\cdot\|$ is semi-monotone, it is clearly regular.

For the converse, suppose that $\|\cdot\|$ is regular. Then

$$
\left\|\left(a_{1}, a_{2}, \ldots, a_{n}\right)\right\| \geq \max _{1 \leq k \leq n}\left\|\left(a_{1}, \ldots, a_{k-1}, 0, a_{k+1}, \ldots, a_{n}\right)\right\|
$$

for all $\left(a_{1}, a_{2}, \ldots, a_{n}\right) \in \mathbb{R}^{n}$. Let $k$ be a positive integer such that $1 \leq k \leq n$, let $\left(a_{1}, a_{2}, \ldots, a_{n}\right) \in \mathbb{R}^{n}$, and let $t \geq 1$. Then we have

$$
\begin{aligned}
& \left\|\left(a_{1}, a_{2}, \ldots, a_{n}\right)\right\| \\
& \begin{array}{r}
\leq\left(1-t^{-1}\right)\left\|\left(a_{1}, \ldots, a_{k-1}, 0, a_{k+1}, \ldots, a_{n}\right)\right\| \\
\quad+t^{-1}\left\|\left(a_{1}, \ldots, a_{k-1}, t a_{k}, a_{k+1}, \ldots, a_{n}\right)\right\|
\end{array} \\
& \leq\left(1-t^{-1}\right)\left\|\left(a_{1}, \ldots, a_{k-1}, t a_{k}, a_{k+1}, \ldots, a_{n}\right)\right\| \\
& \quad+t^{-1}\left\|\left(a_{1}, \ldots, a_{k-1}, t a_{k}, a_{k+1}, \ldots, a_{n}\right)\right\| \\
& =\left\|\left(a_{1}, \ldots, a_{k-1}, t a_{k}, a_{k+1}, \ldots, a_{n}\right)\right\| .
\end{aligned}
$$


Now, let $\left(a_{1}, a_{2}, \ldots, a_{n}\right)$ and $\left(b_{1}, b_{2}, \ldots, b_{n}\right)$ be elements of $\mathbb{R}^{n}$ such that $\left|a_{i}\right| \leq\left|b_{i}\right|$ and $a_{i} b_{i} \geq 0$ for all $i=1,2, \ldots, n$. Then we obtain

$$
\begin{aligned}
\left\|\left(a_{1}, a_{2}, \ldots, a_{n}\right)\right\| & \leq\left\|\left(b_{1}, a_{2}, \ldots, a_{n}\right)\right\| \\
& \leq\left\|\left(b_{1}, b_{2}, a_{3}, \ldots, a_{n}\right)\right\| \\
& \vdots \\
& \leq\left\|\left(b_{1}, b_{2}, \ldots, b_{n}\right)\right\|
\end{aligned}
$$

Thus, the norm $\|\cdot\|$ is semi-monotone.

Therefore, the regularity of norms can be viewed as monotonicity in each quadrants. We now characterize the existence of $B_{2}$-orthonormal bases in terms of regular norm.

Theorem 3.7. Let $X$ be an $n$-dimensional normed linear space. Then, $X$ has a $B_{2}$-orthonormal basis if and only if it is isometrically isomorphic to the space $\mathbb{R}^{n}$ endowed with a regular norm.

Proof. Assume that $X$ has a $B_{2}$-orthonormal basis $\left\{e_{1}, e_{2}, \ldots, e_{n}\right\}$. Then, $X$ is identified with the space $\left(\mathbb{R}^{n},\|\cdot\|_{0}\right)$ under the identification

$$
X \ni \sum_{k=1}^{n} \alpha_{k} e_{k} \longleftrightarrow\left(a_{1}, a_{2}, \ldots, a_{n}\right) \in \mathbb{R}^{n}
$$

where the norm $\|\cdot\|_{0}$ is given by

$$
\left\|\left(a_{1}, a_{2}, \ldots, a_{n}\right)\right\|_{0}=\left\|\sum_{k=1}^{n} a_{k} e_{k}\right\|
$$

for all $\left(a_{1}, a_{2}, \ldots, a_{n}\right) \in \mathbb{R}^{n}$. Moreover, one can obtain that $\|\cdot\|_{0} \in R N_{n}$ since $\left\{e_{1}, e_{2}, \ldots, e_{n}\right\}$ is $B_{2}$-orthonormal.

For the converse, suppose that $X$ is isometrically isomorphic to the space $\mathbb{R}^{n}$ endowed with a regular norm $\|\cdot\|_{0}$. Let $T$ be an isometric isomorphism from $X$ onto $\left(\mathbb{R}^{n},\|\cdot\|_{0}\right)$. For each $i=1,2, \ldots, n$, let $e_{i}$ be the element of $X$ such that

$$
T e_{i}=(0, \ldots, 0, \stackrel{(i)}{1}, 0, \ldots, 0) .
$$

Then, it follows that

$$
\left\|\sum_{k=1}^{n} \alpha_{k} e_{k}\right\|=\left\|\left(\alpha_{1}, \alpha_{2}, \ldots, \alpha_{n}\right)\right\|_{0}
$$


for all $\left(\alpha_{1}, \alpha_{2}, \ldots, \alpha_{n}\right) \in \mathbb{R}^{n}$. Thus,

$$
\begin{aligned}
\left\|\sum_{k=1}^{n} \alpha_{k} e_{k}\right\| & =\left\|\left(\alpha_{1}, \alpha_{2}, \ldots, \alpha_{n}\right)\right\|_{0} \\
& \geq \max _{1 \leq k \leq n}\left\|\left(a_{1}, \ldots, a_{k-1}, 0, a_{k+1}, \ldots, a_{n}\right)\right\|_{0} \\
& =\max _{1 \leq k \leq n}\left\|\sum_{i \neq k} a_{i} e_{i}\right\|
\end{aligned}
$$

holds for all $\left(a_{1}, a_{2}, \ldots, a_{n}\right) \in \mathbb{R}^{n}$, which shows that $\left\{e_{1}, e_{2}, \ldots, e_{n}\right\}$ satisfies $(B 2)$ by Proposition 2.1. The proof is complete.

Remark 3.8. A normal basis $\left\{e_{1}, e_{2}, \ldots, e_{n}\right\}$ for an $n$-dimensional normed linear space $X$ is said to be $R$-orthonormal if it satisfies the following condition:

$(R) e_{k} \perp_{R} M_{k}$ for all $k=1,2, \ldots, n$.

Then, it is easy to check that an $n$-dimensional normed linear space $X$ has an $R$-orthonormal basis if and only if it is isometrically isomorphic to the space $\mathbb{R}^{n}$ endowed with an absolute normalized norm. Thus, we finally have the following relationship.

$$
\begin{aligned}
& (R) \Rightarrow\left(B_{2}\right) \Rightarrow\left(B_{1}\right) \\
& A N_{n} \subset \begin{array}{ccc}
\uparrow & & \uparrow \\
N_{n} & \subset & N N_{n}
\end{array}
\end{aligned}
$$

Acknowledgement. The second author was supported in part by Grants-inAid for Scientific Research (No. 23540189), Japan Society for the Promotion of Science.

\section{REFERENCES}

1. J. Alonso, Any two-dimensional normed space is a generalized Day-James space, J. Inequal. Appl. 2011, 2011:2, 3 pp.

2. J. Alonso, H. Martini and S. Wu, On Birkhoff orthogonality and isosceles orthogonality in normed linear spaces, Aequationes Math. 83 (2012), 153-189.

3. R. Bhatia, Matrix analysis, Springer-Verlag, New York, 1997.

4. G. Birkhoff, Orthogonality in linear metric spaces, Duke Math. J. 1 (1935), 169-172.

5. S.O. Carlsson, Orthogonality in normed linear spaces, Ark. Mat. 4 (1962), 297-318.

6. M.M. Day, Polygons circumscribed about closed convex curves, Trans. Amer. Math. Soc. 62 (1947), 315-319.

7. M.M. Day, Some characterizations of inner product spaces, Trans. Amer. Math. Soc. 62 (1947), 320-337.

8. C.R. Diminnie, A new orthogonality relation for normed linear spaces, Math. Nachr. 114 (1983), 197-203.

9. R.C. James, Orthogonality in normed linear spaces, Duke Math. J. 12 (1945), 291-302.

10. R.C. James, Inner products in normed linear spaces, Bull. Amer. Math. Soc. 53 (1947), $559-566$.

11. R.C. James, Orthogonality and linear functionals in normed linear spaces, Trans. Amer. Math. Soc. 61 (1947), 265-292.

12. R. Khalil, Orthogonality in Banach spaces, Math. J. Toyama Univ. 13 (1990), 185-205.

13. B.D. Roberts, On the geometry of abstract vector spaces, Tohoku Math. J. 39 (1934), 42-59. 
14. K.-S. Saito, M. Kato and Y. Takahashi, Absolute norms on $\mathbb{C}^{n}$, J. Math. Anal. Appl. 252 (2000), 879-905.

15. K. Sundaresan and O.P. Kapoor, T-orthogonality and nonlinear functionals on topological vector spaces, Canad. J. Math. 25 (1973), 1121-1131.

16. R. Tanaka and K.-S. Saito, Every n-dimensional normed space is the space $\mathbb{R}^{n}$ endowed with a normal norm, J. Inequal. Appl. 2012, 2012:284, 5pp.

${ }^{1}$ Department of Mathematical Science, Graduate School of Science and TechNOLOGy, NiIgata University, NiIgata 950-2181, Japan.

E-mail address: ryotarotanaka@m.sc.niigata-u.ac.jp

2 Department of Mathematics, Faculty of Science, Nimgata University, Niigata 950-2181, JAPAN.

E-mail address: saito@math.sc.niigata-u.ac.jp 